\title{
The combination of ApoCIII, hepatic lipase and hormono sensitive lipase gene polymorphisms suggests an association with susceptibility to gestational hypertension
}

\author{
Nathalie Bernard · Joël Girouard · \\ Jean-Claude Forest · Yves Giguère
}

Received: 18 October 2006/ Accepted: 20 December 2006/Published online: 15 February 2007

(C) The Japan Society of Human Genetics and Springer 2007

\begin{abstract}
Dyslipidemia and insulin resistance contribute to the endothelial cell dysfunction in hypertensive disorders of pregnancy (HDP) and increase the long-term risk of cardiovascular disease (CVD). The genes linking susceptibility to gestational hypertension (GH) and/or preeclampsia (PE) to the long-term risk of CVD are still unknown. We evaluated the potential association between 14 polymorphisms from six genes involved in lipid metabolism and insulin action and the risk of HDP: namely the lipoprotein lipase $(L P L)$, hepatic lipase $(L I P C)$, hormone sensitive lipase (LIPE), cholesteryl ester transfer protein (CETP), ApoCIII and ApoE gene polymorphisms. Overall, 169 women with HDP [proteinuria (PE) and gestational hypertension without proteinuria $(\mathrm{GH})]$ and 169 controls matched for age and year of delivery were genotyped. Homozygosity of the $-514 \mathrm{~T}$ allele of the $514 \mathrm{C}>\mathrm{T}$ polymorphism (LIPC gene) decreased the risk of $\mathrm{GH}\left(\mathrm{OR}=0.17, \mathrm{CI}_{95}: 0.02-0.76\right)$, while there were more $-60 \mathrm{G}$ carriers of the $-60 \mathrm{C}>\mathrm{G} L I P E$ gene polymorphism $\left(\mathrm{OR}=3.51, \mathrm{CI}_{95}: 1.02-12.10\right)$ among $\mathrm{GH}$ cases, but not in PE cases. The common ApoCIII two-locus $-482 \mathrm{CC} / 3238 \mathrm{CC}$ genotype was lower in women with $\mathrm{GH}$ compared with controls $(\mathrm{OR}=0.53$,
\end{abstract}

\footnotetext{
N. Bernard · J. Girouard · J.-C. Forest · Y. Giguère ( $₫)$ Unité de recherche en périnatalogie,

Centre de recherche du CHUQ, Hôpital Saint-François

d'Assise, 10, rue de l'Espinay,

G1L 3L5 Quebec, QC, Canada

e-mail: Yves.Giguere@crsfa.ulaval.ca

J. Girouard · J.-C. Forest · Y. Giguère

Département de Biologie médicale,

Faculté de médecine, Université Laval,

Quebec, QC, Canada
}

$\left.\mathrm{CI}_{95}: 0.3-0.9\right)$. The combined frequency of at-risk genotypes was higher in cases of $\mathrm{GH}$ compared with controls [one at-risk genotype: $\mathrm{OR}=3.38(95 \% \mathrm{CI}$ : $0.48-41.8)$; two or more at-risk genotypes: $\mathrm{OR}=7.14$ (95\% CI: $1.21-92.3, P=0.01$ )], suggesting a gene-dose effect. We conclude that the combined effect of $L I P C$, $L I P E$ and ApoCIII gene polymorphisms may increase the likelihood of GH, but seemingly not of PE.

Keywords Association study - Candidate gene analysis - Cardiovascular disease - DNA polymorphisms - Gestational hypertension . Preeclampsia

\section{Introduction}

Hypertensive disorders of pregnancy (HDP) are characterized by high blood pressure $(\geq 140 \mathrm{mmHg} /$ $\geq 90 \mathrm{mmHg}$ ) appearing after 20 weeks of gestation. HDP include hypertension with (preeclampsia, PE) or without (gestational hypertension, $\mathrm{GH}$ ) proteinuria, defined as $>300 \mathrm{mg} / 24 \mathrm{~h}$ (2000; Roberts and Cooper 2001; Roberts et al. 2003; Solomon and Seely 2001; Walker 2000). Overall, these disorders complicate 5$10 \%$ of pregnancies in industrialized countries (Cooper et al. 1993; Roberts and Cooper 2001; Solomon and Seely 2001; Walker 2000), and they represent a major cause of fetal and maternal morbidity and mortality (Chappell et al. 2002; Roberts et al. 2003).

There is evidence suggesting that HDP are multifactorial (Roberts and Cooper 2001; Talosi et al. 2000; Thornton and Macdonald 1999), but the etiology and mechanisms causing PE and GH are still undefined. Current pathophysiological hypotheses imply inade- 
quate trophoblastic invasion, oxidative stress, endothelial dysfunction, inappropriate maternal immunological response and genetic susceptibility (Dekker and Sibai 1998; Roberts and Cooper 2001; Sibai et al. 2005; Solomon and Seely 2001; Talosi et al. 2000). A number of studies have described a strong familial trend to developing HDP (Arngrimsson et al. 1995; Chesley et al. 1968; Chesley and Cooper 1986; Salonen Ros et al. 2000), and recently a heritability estimate of 0.47 was observed for HDP in a large sample of over 2,000 twin pairs of women, indicating that HDP, both with proteinuria $(\mathrm{PE})$ and without proteinuria $(\mathrm{GH})$, is highly heritable (Salonen Ros et al. 2000). Published data suggest a significant genetic component to HDP, which may include fetal and environmental factors (Broughton Pipkin 2001; Chesley and Cooper 1986; Cooper 1993; GOPEC Consortium 2005; Roberts and Cooper 2001; Salonen Ros et al. 2000; Thornton and Macdonald 1999). Finally, it is still debated whether PE and GH represent different manifestations of the same entity or rather distinct pathogenic disorders (Brown et al. 2001; Fisher et al. 1981; Seely and Solomon 2003; Vatten and Skjaerven 2004).

Retrospective studies suggest that HDP are associated with many risk factors contributing to cardiovascular disease (CVD) (Jonsdottir et al. 1995; Roberts 2000). An unfavorable lipid profile associated with an increased CVD risk has also been found in women who suffered from HDP (Forest et al. 2005; Pouta et al. 2004; Roberts 2000; Roberts and Cooper 2001). Triglycerides (TG), free fatty acids (FFA) and low-density lipoprotein cholesterol (LDL-C) concentrations, especially small dense LDL-C, are increased in the presence of HDP, whereas the high-density lipoprotein cholesterol (HDL-C) concentration is decreased (Seely and Solomon 2003). HDP (Chappell et al. 2002; Dekker and Sibai 1998; Roberts 2000; Roberts and Cooper 2001; Seely and Solomon 2003; Solomon and Seely 2001), and GH in particular (Caruso et al. 1999; Roberts et al. 1998), have been shown to be associated with insulin resistance, and women who never developed HDP during pregnancy are at lower risk of CVD (Roberts 2000; Williams 2003; Wilson et al. 2003). Thus, HDP may constitute a first sign of increased CVD risk later in life, possibly through a predisposition to develop the metabolic syndrome that encompasses hypertension, lipid profile alterations, insulin resistance and abdominal obesity (Forest et al. 2005; Pouta et al. 2004; Williams 2003).

Several genetic factors with modest effect probably contribute to $\mathrm{PE}$ and/or GH susceptibility. Potential susceptibility genes for HDP involved in thrombophilia (Factor $V$ Leiden, methylenetetrahydrofolate reductase and prothrombin genes) and blood pressure control (Angiotensinogen gene) have been studied, leading to conflicting results (Belo et al. 2004; GOPEC Consortium 2005; Hubel et al. 1999; Kim et al. 2001; Levesque et al. 2004; Roberts and Cooper 2001; Sibai et al. 2005; Walker 2000). Considering the association of dyslipidemia with both HDP and CVD, variants of genes associated with lipid homeostasis have been investigated in small-scale studies (Belo et al. 2004; Hubel et al. 1999; Kim et al. 2001; Roberts and Cooper 2001; Walker 2000), but need to be further explored. Indeed, only a few studies have investigated the genes involved in lipid homeostasis, and only preeclamptic women were included as cases. Hubel et al. 1999 found an association between the N291S polymorphism of the LPL gene and the risk of PE in American women, but Kim et al. 2001 were not able to confirm such results in Korean women. Makkonen et al. 2001 did not observe any association between $A p o E$ genotypes and the risk of PE in a study of 133 cases and 91 controls. Also, Belo et al. 2004 did not find any associations between ApoE and CETP polymorphism frequencies between 144 controls and 51 preeclamptic women.

We hypothesized that polymorphisms of genes involved in lipid homeostasis and associated with the action of insulin represent good candidates for bridging $\mathrm{GH}$ and/or PE to long-term CVD. In the present study, we aimed to compare the prevalence of 14 polymorphisms from six genes, both individually and jointly, previously associated with lipid metabolism and insulin action, i.e., lipoprotein lipase ( $L P L ;$ D9N, N291S, G188E, P207L, D250N and S447X polymorphisms), hepatic lipase $(L I P C ;-514 \mathrm{C}>\mathrm{T})$, hormone-sensitive lipase $(L I P E, \quad-60 \mathrm{C}>\mathrm{G})$, apolipoprotein CIII (ApoCIII; $-482 \mathrm{C}>\mathrm{T}, 3238 \mathrm{C}>\mathrm{G})$ and E (ApoE; $\mathrm{R} 112 \mathrm{C}, \mathrm{C} 158 \mathrm{R})$ and cholesteryl ester transfer protein (CETP; Taq1B, R451Q) genes in French-Canadian women with or without HDP.

\section{Materials and methods}

\section{Study subjects}

The study sample consisted of 169 cases (106 with GH and 63 with PE) and 169 controls matched for maternal age $( \pm 1$ year $)$ and year of delivery $( \pm 1$ year). They were recruited from a cohort of women studied during pregnancy to identify biological and echographic markers of HDP in nulliparous women (Irion et al. 1998; Masse et al. 1993, 1998). Most subjects were Caucasians (98\%, by self assessment of origin) from the Quebec City metropolitan area where $93 \%$ of the 
population is of French ancestry (Santé Québec 1995). Subjects were classified according to the NHBPEP (National High Blood Pressure Education Program working group) criteria. Briefly, the diagnosis of HDP was defined as a blood pressure of $140 / 90 \mathrm{mmHg}$ or above on two occasions $6 \mathrm{~h}$ apart after 20 weeks of pregnancy, but before labor in women with proven normal blood pressure in the first trimester. Cases of HDP included GH (without proteinuria) and PE [with proteinuria $>300 \mathrm{mg} / 24 \mathrm{~h}$ or a positive qualitative results ("dipstick 2+")]. Blood pressure had to return to normal values $(\leq 120 / 80 \mathrm{mmHg})$ in the post-partum period to be included as cases. Controls had a history of uncomplicated pregnancy. Multiparous women and those with a history of diabetes, renal disease, CVD and those who developed gestational diabetes were excluded. The study was approved by the Hospital's Ethic's Review Board, and all subjects gave informed consent.

\section{Analysis of polymorphisms}

DNA was extracted from whole blood using the standard protocol of the QIAamp 96 Blood Kit (QIAGEN). The DNA was redissolved in Tris-EDTA buffer (10 mM Tris; 1 mM EDTA; $\mathrm{pH} 8$ ) and stored at $-20^{\circ} \mathrm{C}$ until genotyping was performed.

All single nucleotide polymorphisms (SNP) were genotyped by an allele-specific oligonucleotide-polymerase chain reaction (ASO-PCR) method optimized in our laboratory. Oligonucleotide pairs were designed for PCR amplification of each SNP studied with the use of the Oligo 4S software (Table 1). The reactions were carried out in a total volume of $25 \mu \mathrm{l}$ containing $200 \mu \mathrm{M}$ dNTPs, $0.5 \mu \mathrm{M}$ of common primer, $0.5 \mu \mathrm{M}$ of allele-specific primer, 0.4 U Taq DNA polymerase (QIAGEN), 1X QIAGEN PCR Buffer, 1X Q-Solution and 20-40 ng DNA. Negative controls without DNA or Taq DNA polymerase, as well as positive controls for each genotype, were run with each set of amplification. In general, the initial denaturation was performed at $95^{\circ} \mathrm{C}$ for $5 \mathrm{~min}$, followed by $32-35$ cycles of denaturation at $95^{\circ} \mathrm{C}$ for $45 \mathrm{~s}$, primer annealing at an optimized temperature (Table 1) for $45 \mathrm{~s}$, extension at $72^{\circ} \mathrm{C}$ for $45 \mathrm{~s}$ and a final extension at $72^{\circ} \mathrm{C}$ for $7 \mathrm{~min}$. Amplified fragments of each allele were separated on a $1 \%$ agarose gel electrophoresis to determine the genotype. For each polymorphism, genotypes were validated by PCR-endonuclease digestion for a subset of samples, and the concordance rate was $\geq 97 \%$ for each polymorphism. Three independent readers interpreted each polymorphism. Genotypes without a $100 \%$ concordance $(3 / 3)$ were rejected unless a repeat ASO-
PCR reaction resulted in a $100 \%$ concordance. Overall, the concordance rate was $\geq 96 \%$ for each polymorphism studied.

\section{Statistics}

General clinical characteristics between cases and controls were compared using Student's $t$ tests, $\chi^{2}$ tests or Fisher tests where appropriate. Genotype frequencies were tested against Hardy-Weinberg equilibrium (HWE) by $\chi^{2}$ contingency table analysis in the whole study sample, as well as in cases and controls separately. For the two polymorphisms of both ApoCIII and CETP genes, we estimated the coefficient of linkage disequilibrium $D^{\prime}\left(\mathrm{D} / \mathrm{D}_{\max }\right.$ if $\left.\mathrm{D}>0\right) \pm \mathrm{SD}$ and $P$ value for a two-marker combination using the $2 \mathrm{LD}$ program (Zhao et al. 2000). Since allelic frequencies of LPL mutations resulting in a severe decrease in LPL activity were very low, we combined N291S, D9N, P207L, G188E and D250N polymorphisms to increase the power to detect an association (Ishimura-Oka et al. 1992; Zhang et al. 1998).

Genotype frequencies were compared among all cases together $(\mathrm{PE}+\mathrm{GH})$ and separately for $\mathrm{GH}$ and PE subgroups and their controls by contingency tables or by the Fisher's exact probability test where applicable. We performed conditional logistic regression to compute odds ratios (OR) and $95 \%$ confidence intervals (CI) in order to test for differences in the prevalence of at-risk genotypes between cases and controls. The frequency of homozygotes for the common allele was considered as the reference for comparisons $(\mathrm{OR}=1)$. Since the body mass index $(\mathrm{BMI})$ is associated with HDP and some of the genotypes under study, all results were adjusted for the pre-pregnancy BMI. Finally, an OR was computed to evaluate the possibility of a joint multi-locus association with HDP, GH and $\mathrm{PE}$ of polymorphisms individually associated with HDP $(P<0.05)$ by testing the impact of increasing prevalence of these at-risk genotypes $(0,1,2$ and more). According to the results of individual loci, a recessive or dominant model of gene effect was applied. To test the robustness and consistency of statistically significant results, we performed 2,000 permutation-based simulations of genotypes (Efron and Tibshirani 1995). By permuting the genotype labels, we hypothesize that the association does not depend on the genotype. Thus, we obtained 2,000 new data sets with the genotype (carrier status or combined genotype when applicable) randomly rearranged. We referred to the permutation $P$ value as the proportion of permutations out of 2,000 with a $P$ value smaller than or equal to the observed $P$ value. Since the 
Table 1 ASO-PCR primers and annealing temperature for each polymorphism studied

\begin{tabular}{|c|c|c|}
\hline Polymorphisms & ASO-PCR primers $\left(5^{\prime} \rightarrow 3^{\prime}\right)$ & $\begin{array}{l}\text { Annealing } \\
\text { temperature }\end{array}$ \\
\hline$L P L \mathrm{~N} 291 \mathrm{~S}$ & $\begin{array}{l}\text { R-GTTATAGTTTGTTGACCCGAG } \\
\text { F-CTGGGCTATGAGATCAA (A allele) } \\
\text { F-CTGGGCTATGAGATCA } \overline{\mathbf{G}} \text { (G allele) }\end{array}$ & $56.0^{\circ} \mathrm{C}$ \\
\hline$L P L \mathrm{D} 9 \mathrm{~N}$ & $\begin{array}{l}\text { F-AGAAGACAGAATTAACTAAGG } \\
\text { R-AATTTACTTTCGATGT } \text { (G allele) } \\
\text { R-AATTTACTTTCGATGT } \underline{\text { (A allele) }}\end{array}$ & $48.1^{\circ} \mathrm{C}$ \\
\hline$L P L \mathrm{P} 207 \mathrm{~L}$ & 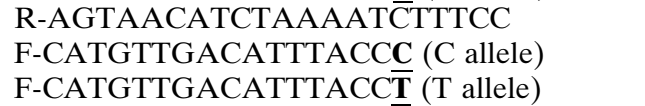 & $51.5^{\circ} \mathrm{C}$ \\
\hline$L P L \mathrm{G} 188 \mathrm{E}$ & $\begin{array}{l}\text { R-GAGGTCAGTGGAGTCTTCCTG } \\
\text { F-CACACATTCACCAGAG } \mathbf{G} \text { (G allele) } \\
\text { F-CACACATTCACCAGAG } \text { (A allele) }\end{array}$ & $52.5^{\circ} \mathrm{C}$ \\
\hline$L P L \mathrm{D} 250 \mathrm{~N}$ & $\begin{array}{l}\text { R-CTCTGTGCTGATAGCCTAGCC } \\
\text { F-TCCATTCATCTCTTCATCG (G allele) } \\
\text { F-TCCATTCATCTCTTCATC } \overline{\text { (A allele) }}\end{array}$ & $50.0^{\circ} \mathrm{C}$ \\
\hline LPL S447X & $\begin{array}{l}\text { F-GCAGATGCTAAGAGATGGCAG } \\
\text { R-GAATGCTCACCAGCCTG (C allele) } \\
\text { R-GAATGCTCACCAGCCT } \overline{\mathbf{C}}(\mathrm{G} \text { allele })\end{array}$ & $57.5^{\circ} \mathrm{C}$ \\
\hline$L I P C-514 \mathrm{C}>\mathrm{T}$ & $\begin{array}{l}\text { F-CTCCAGAAGGTAAGAACCTCC } \\
\text { R-AAAACCCTTCACCCCCG (C allele) } \\
\text { R-AAAACCCTTCACCCCC音 }(\text { T allele })\end{array}$ & $58.0^{\circ} \mathrm{C}$ \\
\hline$L I P E-60 \mathrm{C}>\mathrm{G}$ & $\begin{array}{l}\text { F-CCTTAATCGGCTTTTGTC } \overline{\bar{T} T C C} \\
\text { R-GCCTGCCCAGGGTGTAG (C allele) } \\
\text { R-GCCTGCCCAGGGTGTA } \overline{\mathbf{C}} \text { (G allele) }\end{array}$ & $55.0^{\circ} \mathrm{C}$ \\
\hline ApoCIII 3238C > G (Sst1) & $\begin{array}{l}\text { R-ACTCAAGTCATGTTGTGCAGC } \\
\text { F-TGCCTATCCATCCTGC }(\mathrm{C} \text { allele }) \\
\text { F-TGCCTATCCATCCTGC } \overline{\mathbf{G}}(\mathrm{G} \text { allele })\end{array}$ & $57.7^{\circ} \mathrm{C}$ \\
\hline Apo CIII $-482 \mathrm{C}>\mathrm{T}$ & $\begin{array}{l}\text { R-GCTGACCAGTGGAGATGAGGG } \\
\text { F-CGGAGCCACTGATGCCC (C allele) } \\
\text { F-CGGAGCCACTGATGCC } \overline{\mathbf{T}}(\mathrm{T} \text { allele })\end{array}$ & $62.0^{\circ} \mathrm{C}$ \\
\hline ApoE R158C & $\begin{array}{l}\text { R-GGCTCGAACCAGCTCTT̄GAGG } \\
\text { F-CGATGACCTGCAGAAG } \mathbf{C}(\mathrm{C} \text { allele }) \\
\text { F-CCGATGACCTGCAGAAĞ } \underline{\mathbf{T}}(\mathrm{T} \text { allele })\end{array}$ & $59.3^{\circ} \mathrm{C}$ \\
\hline ApoE C112R & $\begin{array}{l}\text { F-CTCATCCCCATCTCGCCC } \overline{G C C C C} \\
\text { R-TACTGCACCAGGCGGCCGCA }(\mathrm{T} \text { allele }) \\
\text { R-TACTGCACCAGGCGGCCGC } \overline{\mathbf{G}}(\mathrm{C} \text { allele })\end{array}$ & $69.7^{\circ} \mathrm{C}$ \\
\hline CETP R451Q & $\begin{array}{l}\text { R-TTGGAGACTTCTAGCTCAAGC } \\
\text { F-CCTGAGATTATCACTCA (A allele) } \\
\text { F-CCTGAGATTATCACTC } \overline{\mathbf{G}}(\mathrm{G} \text { allele })\end{array}$ & $\begin{array}{l}47.5^{\circ} \mathrm{C} \\
50.5^{\circ} \mathrm{C}\end{array}$ \\
\hline CETP Taq1B & $\begin{array}{l}\text { F-ACACCACTGCCTGATA } \bar{A} \text { CCAT } \\
\text { R-GATCTGAACCCTAACT } \mathbf{C}(\mathrm{G} \text { allele) } \\
\text { R-GATCTGAACCCTAACT } \underline{\mathbf{T}} \text { (A allele) }\end{array}$ & $50.2^{\circ} \mathrm{C}$ \\
\hline
\end{tabular}

$L P L$ lipoprotein lipase gene; $L I P C$ hepatic lipase gene; LIPE hormone-sensitive lipase gene; ApoCIII apolipoprotein CIII gene; ApoE apolipoprotein E gene; CETP cholesteryl ester transfer protein gene delivery was lower. Although within normal range, systolic blood pressure was already higher during the first trimester in women who developed HDP. A family history of hypertension was significantly more frequent in these cases. Smoking was more prevalent among controls.

Hardy-Weinberg equilibrium

In the whole study sample, all (one-locus) genotype frequencies were in Hardy-Weinberg equilibrium (HWE). When $\chi^{2}$ contingency table analysis was carried out only in women with $\mathrm{GH}$, the $-482 \mathrm{C}>\mathrm{T}$ polymorphism of the ApoCIII gene did not follow HWE $(P=0.01)$. Also, in the whole study group, the ob-
Table 2 summarizes the general characteristics of the study population. Pre-pregnancy BMI, weight gain and systolic and diastolic blood pressures were significantly higher in women who developed HDP compared with matched controls, whereas the gestational age at 
Table 2 General characteristics of the study population

\begin{tabular}{|c|c|c|c|c|c|c|}
\hline Variables & $\begin{array}{l}\text { Controls } \\
(n=169)\end{array}$ & $\begin{array}{l}\text { HDP } \\
(n=169)\end{array}$ & $\begin{array}{l}\text { Controls } \\
(n=106)\end{array}$ & $\begin{array}{l}\mathrm{GH} \\
(n=106)\end{array}$ & $\begin{array}{l}\text { Controls } \\
(n=63)\end{array}$ & $\begin{array}{l}\mathrm{PE} \\
(n=63)\end{array}$ \\
\hline Age at delivery (years) & $27 \pm 4^{\mathrm{a}}$ & $27 \pm 4$ & $27 \pm 4$ & $27 \pm 4$ & $27 \pm 4$ & $27 \pm 4$ \\
\hline Pre-pregnancy BMI $\left(\mathrm{kg} / \mathrm{m}^{2}\right)$ & $21.9 \pm 3.3$ & $24.1 \pm 4.3^{*}$ & $21.7 \pm 3.0$ & $24.6 \pm 4.4^{*}$ & $22.1 \pm 3.7$ & $23.3 \pm 4.1$ \\
\hline Weight gain (kg) & $16.3 \pm 5.7$ & $19.7 \pm 7.7^{*}$ & $16.2 \pm 5.7$ & $20.2 \pm 8.1 *$ & $16.4 \pm 5.8$ & $19.0 \pm 6.9 * *$ \\
\hline Weeks gestation at delivery & $39.4 \pm 2.1$ & $38.8 \pm 2.3^{* *}$ & $39.2 \pm 2.3$ & $39.1 \pm 2.0$ & $39.6 \pm 1.6$ & $38.3 \pm 2.7^{*}$ \\
\hline Baby's weight $(\mathrm{g})$ & $3,335.2 \pm 584.9$ & $3,310.6 \pm 605.2$ & $3,301.5 \pm 611.3$ & $3,423.9 \pm 527.9$ & $3,391.4 \pm 538.2$ & $3,119.8 \pm 679.5^{* *}$ \\
\hline \multirow{2}{*}{\multicolumn{7}{|c|}{$\begin{array}{l}\text { Blood pressure }(\mathrm{mmHg}) \\
\text { Systolic }\end{array}$}} \\
\hline \multicolumn{6}{|l|}{ Systolic } & \\
\hline $9-12$ th w & $113.0 \pm 8.3$ & $119.5 \pm 9.8^{*}$ & $112.6 \pm 8.8$ & $119.9 \pm 10.0^{*}$ & $114.1 \pm 7.2$ & $118.5 \pm 9.5 * *$ \\
\hline 14-18th w & $112.6 \pm 8.8$ & $119.9 \pm 11.1^{*}$ & $112.3 \pm 8.0$ & $120.2 \pm 11.4^{*}$ & $113.0 \pm 10.3$ & $119.2 \pm 10.4^{*}$ \\
\hline 26-28th w & $114.9 \pm 7.4$ & $122.3 \pm 9.1 *$ & $115.0 \pm 7.0$ & $122.4 \pm 9.0^{*}$ & $114.9 \pm 8.2$ & $122.1 \pm 9.4^{*}$ \\
\hline \multicolumn{7}{|l|}{ Diastolic } \\
\hline $9-12$ th w & $65.1 \pm 7.1$ & $66.9 \pm 9.0$ & $64.7 \pm 7.4$ & $66.4 \pm 9.6$ & $65.8 \pm 6.7$ & $67.7 \pm 7.8$ \\
\hline 14-16th w & $62.6 \pm 7.6$ & $66.6 \pm 8.3^{*}$ & $63.0 \pm 8.1$ & $66.7 \pm 8.8^{*}$ & $61.8 \pm 6.7$ & $66.5 \pm 7.5^{*}$ \\
\hline 26-28th w & $65.0 \pm 7.5$ & $68.6 \pm 9.2 *$ & $65.6 \pm 7.7$ & $68.7 \pm 9.1 * *$ & $63.9 \pm 7.1$ & $68.4 \pm 9.4 * *$ \\
\hline Family history of PE (\%) & 4.8 & 6.2 & $5(4.9)$ & 5.8 & 4.8 & 6.4 \\
\hline Family history of HDP (\%) & 29.7 & $48.2 *$ & 31.1 & $51.5^{*}$ & 27.0 & 39.7 \\
\hline Smoking $^{\mathrm{b}}(\%)$ & 28.4 & $16.0^{*}$ & 31.1 & $17.9 * *$ & 23.8 & 12.7 \\
\hline Alcohol consumption ${ }^{\mathrm{c}}(\%)$ & 3.0 & 1.2 & 3.8 & 1.9 & 1.6 & 0 \\
\hline
\end{tabular}

HDP diagnosed as a BP $>140 / 90 \mathrm{mmHg}$ after the 20th week of gestation returning to normal pressure in the postpartum period. There were 106 case control pairs in the gestational hypertension subgroup, and 63 case-control pairs in the preeclampsia subgroup (proteinuria $>300 \mathrm{mg} / 24 \mathrm{~h}$ or $2+$ dipstick in cases)

$B M I$ body mass index; HDP hypertensive disorders in pregnancy; $P E$ preeclampsia; $H B P$ high blood pressure

$* P<0.01 ; * * P<0.05$ ( $P$ value based on two-sample Student's $t$ test for continuous variables and $\chi^{2}$ test for ordinal variables)

a Values are given as means $\pm \mathrm{SD}$

b More than one cigarette/day during pregnancy

c More than one consumption/day during pregnancy

served two-locus ApoCIII $-482 \mathrm{C}>\mathrm{T}$ and $3238 \mathrm{C}>\mathrm{G}$ polymorphisms were in $\mathrm{LD}$, resulting in an excess of double heterozygotes (Table 3).

Table 3 Two-locus Hardy-Weinberg and linkage disequilibrium analyses of the combined $-482 \mathrm{C}>\mathrm{T}$ and $3238 \mathrm{C}>\mathrm{G}$ ApoCIII genotype of the study population

\begin{tabular}{|c|c|c|c|c|c|}
\hline & & -locus ge & pes: $\mathrm{Ob}$ & Expe & \\
\hline & & & $238 \mathrm{C}>\mathrm{C}$ & & \\
\hline & & $\mathrm{CC}$ & CG & GG & \\
\hline & $\mathrm{CC}$ & $182 / 154$ & $2 / 35$ & $1 / 2$ & $186 / 191$ \\
\hline 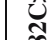 & CT & 79/97 & $51 / 22$ & $1 / 1$ & $131 / 120$ \\
\hline & TT & $6 / 15$ & $5 / 4$ & $3 / 0$ & $14 / 19$ \\
\hline & & $267 / 266$ & $58 / 61$ & $5 / 3$ & $330^{\mathrm{a}}$ \\
\hline
\end{tabular}

$D$ ' coefficient of linkage disequilibrium: $0.88516 ;{ }^{c} p$ value $<\mathbf{0 . 0 0 0 1}$

ApoCIII apolipoprotein CIII gene; D' coefficient of linkage disequilibrium

${ }^{a}$ Observed/expected genotypes according to allele frequencies of the $-482 \mathrm{C}>\mathrm{T}$ and $3238 \mathrm{C}>\mathrm{G}$ polymorphisms

b Sum of controls and cases

c $P$ value determined from 2 LD program (Zhao et al. 2000)
$L P L, L I P C$ and $L I P E$ gene polymorphisms

\section{LPL genotypes}

The carrier frequency of the combined $L P L$ rare mutations (N291S, D9N, P207L, G188E and D250N) did not differ between controls and women with HDP (9.8 vs. $8.0 \%, P>0.05)$ (Table 4$)$. There were no homozygotes and no compound LPL heterozygotes in our study sample. The S447X variant, associated with an increased enzymatic activity and a putative protective effect on lipid homeostasis for $\mathrm{G}$ allele carriers (Skoglund-Andersson et al. 2003; van Bockxmeer et al. 2001), was analyzed alone, and there were no differences between controls (allele frequency $=12.1 \%$ ) and cases (allele frequency $=10.2 \%)($ Table 4$)$.

\section{LIPC genotype}

The rare allele frequency of the $-514 \mathrm{C}>\mathrm{T}$ polymorphism of the LIPC gene did not differ between controls and cases of HDP ( 25.9 vs. $21.3 \%, P>0.05)$, and the proportion of the three genotypes was not significantly different (Table 4). However, in the GH subgroup, the proportion of $-514 \mathrm{~T}$ homozygotes was 
Table 4 Lipoprotein, hepatic and hormone-sensitive lipase gene polymorphisms between cases and controls

\begin{tabular}{|c|c|c|c|c|c|c|}
\hline \multirow[t]{2}{*}{ Polymorphisms } & \multicolumn{3}{|c|}{ Whole study sample } & \multicolumn{3}{|c|}{$\mathrm{GH}$ and controls } \\
\hline & $\begin{array}{l}\text { Controls } \\
(n=169)\end{array}$ & $\begin{array}{l}\text { Cases } \\
(n=169)\end{array}$ & $\begin{array}{l}\text { Odds ratio } \\
\left(\mathrm{CI}_{95}\right)^{\mathrm{a}}\end{array}$ & $\begin{array}{l}\text { Controls } \\
(n=106)\end{array}$ & $\begin{array}{l}\text { Cases } \\
(n=106)\end{array}$ & $\begin{array}{l}\text { Odds ratio } \\
\left(\mathrm{CI}_{95}\right)^{\mathrm{a}}\end{array}$ \\
\hline \multicolumn{7}{|l|}{$L P L$} \\
\hline Non-carriers & 146 & 152 & 1 & 91 & 96 & 1 \\
\hline Carriers $^{\mathrm{b}}$ & 16 & 13 & $0.89(0.39-2.00)$ & 11 & 9 & $0.90(0.31-1.96)$ \\
\hline \multicolumn{7}{|l|}{ LPL S447X } \\
\hline $\mathrm{CC}$ & 133 & 136 & 1 & 83 & 86 & 1 \\
\hline $\mathrm{CG}$ & 31 & 28 & $0.84(0.43-1.66)$ & 20 & 17 & $0.72(0.29-1.78)$ \\
\hline GG & 5 & 3 & $0.46(0.10-2.08)$ & 3 & 3 & $0.82(0.10-7.12)$ \\
\hline \multicolumn{7}{|l|}{$L I P C-514 \mathrm{C}>\mathrm{T}$} \\
\hline $\mathrm{CC}$ & 94 & 105 & 1 & 56 & 63 & 1 \\
\hline $\mathrm{CT}$ & 62 & 56 & $0.96(0.60-1.55)$ & 37 & 40 & $1.01(0.57-1.75)$ \\
\hline $\mathrm{TT}$ & 13 & 8 & $0.62(0.23-1.73)$ & 13 & 3 & $0.17(0.02-0.76)^{*}$ \\
\hline \multicolumn{7}{|l|}{$L I P E-60 \mathrm{C}>\mathrm{G}$} \\
\hline $\mathrm{CC}$ & 156 & 148 & 1 & 101 & 93 & 1 \\
\hline $\mathrm{CG}$ & 12 & 19 & $1.77(0.81-3.89)$ & 4 & 12 & $3.51(1.02-12.10) * *$ \\
\hline GG & 0 & 0 & NA & 0 & 0 & NA \\
\hline
\end{tabular}

GH gestational hypertension; $L P L$ lipoprotein lipase gene; $L I P C$ hepatic lipase gene; $L I P E$ hormone-sensitive lipase gene; NA not applicable

$* P<0.01 ; * * P<0.05$

a Conditional logistic regression and $95 \%$ confidence intervals adjusted for pre-pregnancy body mass index

b Combined genotype for N291S, D9N, P207L, G188E and D250N. The frequency of homozygotes for the common allele was considered as the reference for comparisons $(\mathrm{OR}=1)$

Permutation $P$ value $<0.05$ shown in bold.

decreased five-fold in cases compared to controls (OR: 0.17; $\mathrm{CI}_{95}: 0.02-0.76 ; P=0.01$ )(Table 4), suggesting a protective effect of the $-514 \mathrm{TT}$ genotype.

\section{LIPE genotype}

The rare allele frequency of the $-60 \mathrm{C}>\mathrm{G}$ polymorphism of the LIPE gene did not differ between controls and cases of HDP (3.6 vs. $5.7 \%, P>0.05$ ), and the proportion of the common $-60 \mathrm{C}$ homozygotes and $60 \mathrm{CG}$ heterozygotes (there were no rare $-60 \mathrm{GG}$ homozygotes) was not significantly different (Table 4). However, in the GH subgroup, the proportion of heterozygotes was significantly increased in cases compared to controls (OR: $3.51 ; \mathrm{CI}_{95}: 1.02-12.14 ; P=0.03$ ) (Table 4), suggesting that the rare $-60 \mathrm{G}$ allele of the LIPE gene may be a GH-predisposing gene.

ApoCIII, ApoE and CETP gene polymorphisms

\section{ApoCIII and ApoE genotypes}

The rare allele frequencies between controls and cases of HDP were similar for the $-482 \mathrm{C}>\mathrm{T}(22.4 \mathrm{vs}$. $26.5 \%, P>0.05)$ and $3238 \mathrm{C}>\mathrm{G}(9.2$ vs. $11.5 \%$, $P>0.05)$ variants of the ApoCIII gene, and there were no differences in the proportion of genotypes when both ApoCIII polymorphisms were analyzed individually (Table 5). The $-482 \mathrm{C}>\mathrm{T}$ and $3238 \mathrm{C}>\mathrm{G}$ polymorphisms were in strong linkage disequilibrium $\left(D^{\prime}=0.885, \quad P<0.0001\right.$; Table 3$)$. The common $482 \mathrm{CC} / 3238 \mathrm{CC}$ two-locus genotype, the most frequent two-locus genotype of the $-482 \mathrm{C}>\mathrm{T}$ and $3238 \mathrm{C}>\mathrm{G}$ variants, was more frequent in controls compared with those who developed GH $(60.0 \%$ vs. $43.8 \% ; P=0.01)$ (Table 6). The OR for combined non-CC/CC genotypes was $1.88\left(\mathrm{CI}_{95}: 1.10-3.20\right)$ in the $\mathrm{GH}$ subgroup, suggesting a protective effect of the frequent two-locus CC/CC genotype. Two-locus comparison between all HDP cases and their controls showed a non-significant trend (OR: $1.51 ; \mathrm{CI}_{95}$ : 0.99-2.36, $P=0.054$ ). Finally, the analysis of $A p o E$ variants was negative (Table 5).

\section{CETP genotypes}

The rare allele frequencies between controls and cases were similar for the TaqIB (42.6. vs. $47.6 \%, P>0.05$ ) and R451Q (3.6 vs. $2.7 \%, P>0.05$ ) variants of the $C E T P$ gene, and there were no differences in the proportion of genotypes when both CETP polymorphisms were analyzed individually (Table 5). The TaqIB and R451Q polymorphisms were not in linkage 
Table 5 Apolipoprotein CIII, apolipoprotein E and cholesteryl ester transfer protein gene polymorphisms between cases and controls

\begin{tabular}{|c|c|c|c|c|c|c|}
\hline \multirow[t]{2}{*}{ Polymorphisms } & \multicolumn{3}{|c|}{ Whole study sample } & \multicolumn{3}{|c|}{$\mathrm{GH}$ and controls } \\
\hline & $\begin{array}{l}\text { Controls } \\
(n=169)\end{array}$ & $\begin{array}{l}\text { Cases } \\
(n=169)\end{array}$ & $\begin{array}{l}\text { Odds ratio } \\
\left(\mathrm{CI}_{95}\right)^{\mathrm{a}}\end{array}$ & $\begin{array}{l}\text { Controls } \\
(n=106)\end{array}$ & $\begin{array}{l}\text { Cases } \\
(n=106)\end{array}$ & $\begin{array}{l}\text { Odds ratio } \\
\left(\mathrm{CI}_{95}\right)^{\mathrm{a}}\end{array}$ \\
\hline \multicolumn{7}{|c|}{ ApoCIII $-482 \mathrm{C}>\mathrm{T}$} \\
\hline $\mathrm{CC}$ & 99 & 86 & 1 & 63 & 50 & 1 \\
\hline CT & 58 & 75 & $1.41(0.90-2.30)$ & 37 & 53 & $1.78(1.03-3.08) *$ \\
\hline TT & 8 & 7 & $0.98(0.31-3.10)$ & 5 & 3 & $0.69(0.16-2.92)$ \\
\hline \multicolumn{7}{|c|}{ АроСIII 3238C > G } \\
\hline $\mathrm{CC}$ & 140 & 129 & 1 & 90 & 81 & 1 \\
\hline $\mathrm{CG}$ & 25 & 34 & $1.30(0.70-2.42)$ & 12 & 21 & $1.77(0.85-3.71)$ \\
\hline GG & 3 & 2 & $0.95(0.14-6.57)$ & 3 & 1 & $0.42(0.04-3.70)$ \\
\hline \multicolumn{7}{|l|}{ ApoE } \\
\hline$\varepsilon 3 / \varepsilon 3$ & 86 & 99 & 1 & 52 & 54 & 1 \\
\hline$\varepsilon 2 / \varepsilon 2$ & 1 & 0 & NA & 1 & 0 & NA \\
\hline$\varepsilon 2 / \varepsilon 3$ & 31 & 26 & $0.77(0.38-1.56)$ & 19 & 18 & $0.97(0.36-2.58)$ \\
\hline$\varepsilon 2 / \varepsilon 4$ & 5 & 4 & $0.75(0.18-3.01)$ & 2 & 4 & $2.28(0.36-14.38)$ \\
\hline$\varepsilon 3 / \varepsilon 4$ & 42 & 34 & $0.67(0.37-1.19)$ & 28 & 25 & $0.90(0.43-1.88)$ \\
\hline$\varepsilon 4 / \varepsilon 4$ & 2 & 3 & $1.94(0.30-12.42)$ & 2 & 3 & $2.26(0.35-14.55)$ \\
\hline \multicolumn{7}{|l|}{ CETP TaqIB } \\
\hline GG & 61 & 47 & 1 & 43 & 30 & 1 \\
\hline GA & 72 & 82 & $1.56(0.91-2.65)$ & 43 & 53 & $1.76(0.90-3.41)$ \\
\hline AA & 36 & 39 & $1.28(0.69-2.38)$ & 20 & 23 & $1.42(0.62-3.25)$ \\
\hline \multicolumn{7}{|l|}{ CETP R451Q } \\
\hline GG & 158 & 160 & 1 & 99 & 102 & 1 \\
\hline GA & 10 & 9 & $1.03(0.39-2.70)$ & 6 & 4 & $0.54(0.14-2.14)$ \\
\hline AA & 1 & 0 & NA & 1 & 0 & NA \\
\hline
\end{tabular}

$G H$ gestational hypertension; ApoCIII apolipoprotein CIII gene; ApoE apolipoprotein E gene; CETP cholesteryl ester transfer protein gene; $N A$ not applicable

${ }^{a}$ Conditional logistic regression and $95 \%$ confidence intervals adjusted for pre-pregnancy body mass index. The frequency of homozygotes for the common allele was considered as the reference for comparisons $(\mathrm{OR}=1)$. *Permutation $P$ value $<0.05$ shown in bold

Table 6 Combined two-locus C-482T and C3238G ApoCIII genotypes between cases and controls

\begin{tabular}{|c|c|c|c|c|c|c|}
\hline \multirow[t]{2}{*}{ Genotypes } & \multicolumn{3}{|c|}{ Whole study sample } & \multicolumn{3}{|l|}{$\mathrm{GH}$} \\
\hline & $\begin{array}{l}\text { Controls } \\
(n=169)\end{array}$ & $\begin{array}{l}\text { Cases } \\
(n=169)\end{array}$ & $\begin{array}{l}\text { Odds ratio }\left(\mathrm{CI}_{95}\right)^{\mathrm{a}} \\
P \text { value }^{\mathrm{b}}\end{array}$ & $\begin{array}{l}\text { Controls } \\
(n=106)\end{array}$ & $\begin{array}{l}\text { Cases } \\
(n=106)\end{array}$ & $\begin{array}{l}\text { Odds ratio }\left(\mathrm{CI}_{95}\right)^{\mathrm{a}} \\
P \text { value }^{\mathrm{b}}\end{array}$ \\
\hline \multicolumn{7}{|c|}{ АроСIII $(-482 \mathrm{C}>\mathrm{T} / 3238 \mathrm{C}>\mathrm{G})$} \\
\hline $\mathrm{CC} / \mathrm{CC}$ genotype & 99 & 83 & 1 & 63 & 46 & 1 \\
\hline Other genotypes & 67 & 85 & $\begin{array}{l}1.51(0.99-2.36) \\
P=0.054\end{array}$ & 42 & 59 & $\begin{array}{l}\mathbf{1 . 8 8}(\mathbf{1 . 1 0 - 3 . 2 0 )} \\
P=0.01\end{array}$ \\
\hline
\end{tabular}

GH gestational hypertension; ApoCIII apolipoprotein CIII gene

${ }^{a}$ Conditional logistic regression and $95 \%$ confidence intervals adjusted for pre-pregnancy body mass index

b The frequency of double homozygotes for the common allele was considered as the reference for comparisons $(\mathrm{OR}=1)$. Permutation $P$ value $<0.05$ shown in bold

disequilibrium ( $D^{\prime}: 0.311 ; P=0.11$, data not shown), and two-locus haplotype analysis was non-contributory (data not shown).

\section{Combined frequency of at-risk genotypes}

We calculated the combined frequency of at-risk genotypes of polymorphisms that showed a positive association in univariate analyses [assigning the value
"0" to $-482 \mathrm{CC} / 3238 \mathrm{CC}$ (ApoCIII), -514TT (LIPC) and $-60 C C$ ( $L I P E)$ genotypes, and the value " 1 " to other genotypes, i.e., presence of an at-risk genotype] (Fig. 1a). In the whole study sample, the combined frequency of at-risk genotypes was higher in cases compared to controls, but the relationship was not significant. However, in the GH subgroup, the presence of one or two and more at-risk genotypes resulted in ORs of $3.4\left(\mathrm{CI}_{95}: 0.5-41.8 ; P=0.28\right)$ and $7.1\left(\mathrm{CI}_{95}\right.$ : 

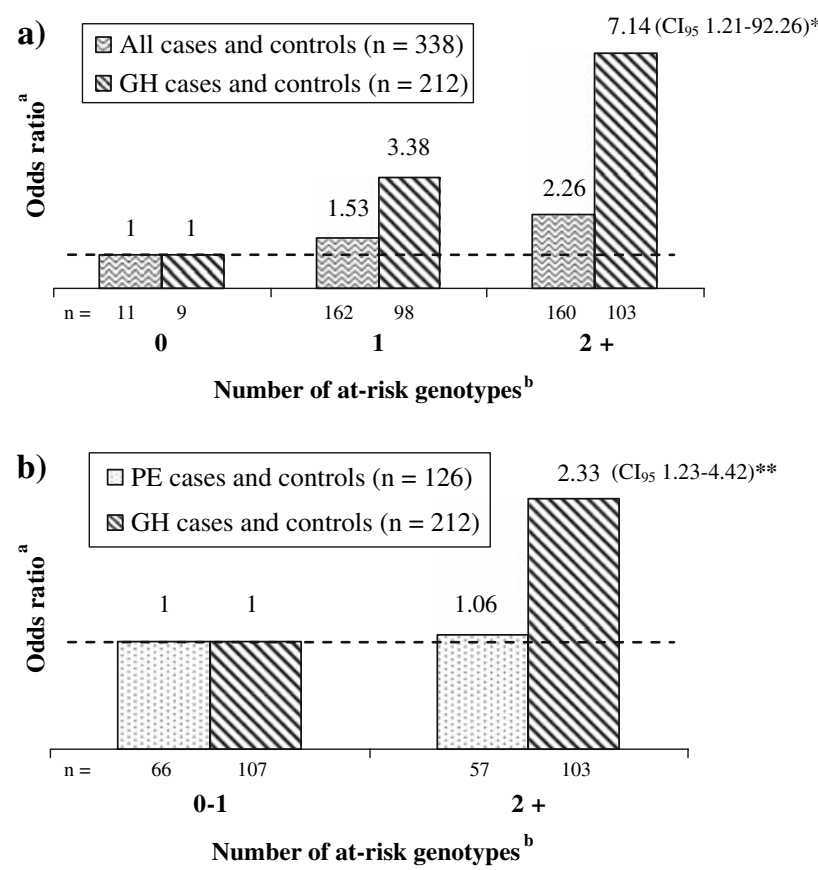

Fig. 1 Risk of HDP, GH and PE according to the number of atrisk genotypes. a Risk of HDP and GH in women with 1 or 2 and more $(2+)$ genotypes compared with those without any at-risk genotype $(0)$ as the reference for comparisons $(\mathrm{OR}=1)$. b Risk of $\mathrm{PE}$ and $\mathrm{GH}$ in women with 2 and more $(2+)$ genotypes compared with those carrying 0 or $1(0-1)$ at-risk genotypes as the reference for comparisons $(\mathrm{OR}=1)$. The number of subjects within each at-risk genotype subgroup is indicated below each histogram. Since the number of PE and matched controls was too low in the ' 0 ' at-risk genotype category $(n=2)$, OR in this subgroup was calculated only after comparing $0-1$ versus $2+$ atrisk genotype categories (Fig. 1b). ${ }^{a}$ Odds ratios after conditional logistic regression adjusted for body mass index. *Permutation $P$ value $=0.01 ; * *$ Permutation $P$ value $=0.004(2,000$ permutations were performed). ${ }^{\mathrm{b}}-482 \mathrm{CC} / 3238 \mathrm{CC}$ (ApoCIII), -514TT (LIPC) and -60CC (LIPE) genotypes: 0; otherwise 1 (other genotypes, i.e., presence of an at-risk genotype). HDP hypertensive disorders of pregnancy; $G H$ gestational hypertension; $P E$ preeclampsia

1.21-92.3; $P=0.01$ ), respectively. Because of the number of PE cases and matched controls in the " 0 " at-risk genotype category $(n=2)$, it was not possible to test for associations in this subgroup. To allow for more robust comparisons between at-risk genotype categories for both $\mathrm{GH}$ and PE subgroups, we tested potential associations between the combined categories " 0 or 1 " vs. " 2 or more (2+)" at-risk genotypes (Fig. 1b). This resulted in about $50 \%$ of subjects per at-risk genotype category. In the GH subgroup, although the OR was, as expected, decreased compared with the previous at-risk genotype comparison model, the presence of $2+$ at-risk genotypes was significantly associated with the presence of a pregnancy complicated by $\mathrm{GH}\left(\mathrm{OR}=2.33 ; \mathrm{CI}_{95}: 1.23-4.42 ; P=0.004\right)$, while there was no trend in the PE-controls subgroup $\left(\mathrm{OR}=1.06 ; \mathrm{CI}_{95}: 0.51-2.22 ; P=0.89\right)$.

\section{Discussion}

The pathogenesis of GH and PE is still undefined, and it is unknown if they represent different manifestations of the same entity or different disorders. There is a growing body of evidence suggesting that exaggerated lipid perturbations and abnormal insulin homeostasis, namely increased blood triglycerides, decreased HDL cholesterol and the presence of atherogenic small, dense LDL-cholesterol more susceptible to oxidation, favor a state of increased oxidative stress contributing to the development of HDP in predisposed women (Hubel et al. 1996; Solomon and Seely 2001; Williams 2003). In this study, we investigated the association between polymorphisms of genes involved in lipid homeostasis and insulin action and HDP, GH and/or $\mathrm{PE}$ in a sample of unrelated French-Canadian women. By studying these candidate genes, we were aiming to bridge genetic susceptibility to HDP with the potential long-term risk of CVD in these women.

In the GH subgroup, significant differences were observed. We found a fourfold decrease in the frequency of LIPC -514T homozygotes in cases compared with controls (Table 4). Although the role of the hepatic lipase activity and variation of the LIPC gene, which bears an insulin-responsive element (IRE) in its promoter region, in CVD risk is controversial and possibly modulated by the metabolic context, environmental factors and genetic background, some data suggest that increased activity of the HL enzyme is related to an atherogenic lipoprotein profile (Deeb et al. 2003). The $514 \mathrm{~T}$ allele is associated with a $30 \%$ reduction of enzymatic activity (Tahvanainen et al. 1998; Zambon et al. 1998), decreased triglycerides, increased HDL cholesterol, reduced formation of small, dense LDL cholesterol (Chen et al. 2003; Deeb and Peng 2000) and insulin resistance in healthy young males (Jansen et al. 2001). Also, we observed a threefold increased frequency of $-60 \mathrm{G}$ carriers of the LIPE gene (Table 4) in the GH group compared with controls. HSL catalyses the rate-limiting step of adipose tissue lipolysis, and it modulates FFA release by adipocytes (Garenc et al. 2002; Pihlajamaki et al. 2001), insulin action and serum lipid levels (Klannemark et al. 1998; Pihlajamaki et al. 2001). It has been suggested that the $-60 \mathrm{C}>\mathrm{G}$ promoter polymorphism of the LIPE gene, which is associated with a $40 \%$ variation in promoter activity in vitro (Talmud et al. 1998), may affect the cholesterol level and insulin sensitivity, but the phenotypic effect of this 
polymorphism is still unclear and probably depends on metabolic homeostasis, body fat distribution and gender (Pihlajamaki et al. 2001). In the PE-only subgroup, however, we did not find any significant differences compared with controls.

We studied the $-482 \mathrm{C}>\mathrm{T}$ and $3238 \mathrm{C}>\mathrm{G}$ polymorphisms of the ApoCIII gene both separately and in combination. The rare alleles of the $-482 \mathrm{C}>\mathrm{T}$ and 3238C > G polymorphisms are considered at-risk genotypes because of their association with dyslipidemia. ApoCIII is a non-competitive LPL inhibitor, and ApoCIII gene polymorphisms can prevent an insulin effect on ApoCIII gene promoter, resulting in inappropriate LPL inhibition and increased plasma TG levels (Talmud and Humphries 1997). In the control group, the $-482 \mathrm{C}>\mathrm{T}$ and $3238 \mathrm{C}>\mathrm{G}$ allele frequencies were similar to what was recently reported in French Canadians, with the $-482 \mathrm{C}>\mathrm{T}$ frequency being lower than in many other Caucasian populations (Garenc et al. 2004). Interestingly, we found more cases of GH carrying the dyslipidemia-associated allele at both loci $(-482 \mathrm{~T}$ allele carriers, $\mathrm{OR}=1.7 ; 3238 \mathrm{G}$ allele carriers, $\mathrm{OR}=1.6$ ), suggesting that women homozygous for both frequent "protecting" $-482 \mathrm{CC} /$ 3238CC ApoCIII genotypes were less susceptible to $\mathrm{GH}$. Indeed, the frequency of the protective $-482 \mathrm{CC} /$ $3238 \mathrm{CC}$ two-loci genotype was significantly lower in women with $\mathrm{GH}$, resulting in an $\mathrm{OR}=1.88$ for those bearing the other two-loci genotypes (Table 6). It is noteworthy that by combining the polymorphisms of three independent genes (LIPC, LIPE and ApoCIII) that showed single gene association, we observed that the risk of GH was additive, while there was no trend in the PE subgroup (Fig. 1).

It is also noteworthy that the ApoCIII $-482 \mathrm{C}>\mathrm{T}$ and $3238 \mathrm{C}>\mathrm{G}$ polymorphisms were in strong linkage disequilibrium $\left(D^{\prime}=0.89 ; P<0.0001\right)$, as was recently observed in French Canadians $\left(D^{\prime}=0.80 ; P<0.0001\right)$ (Garenc et al. 2004). In the present study, the frequency of ApoCIII -482C > T and 3238C > G double heterozygotes was about 2.5 -fold higher than expected (15.5\% vs. $6.7 \%$; Table 3 ). From a study of unrelated adult Caucasians (Dammerman et al. 1993), we determined that the ApoCIII $-482 \mathrm{C}>\mathrm{T}$ and $3238 \mathrm{C}>\mathrm{G}$ polymorphisms were also in $\mathrm{LD}$, resulting in a twofold increase of double heterozygotes $(11.5 \%$ of double heterozygotes observed vs. $5.1 \%$ expected). The molecular basis of the observed excess of double heterozygotes for this two-locus ApoCIII genotype suggests a selective advantage for double heterozygous carriers and needs to be further explored.

Allelic association studies represent a powerful approach to investigate the genetics of complex traits, but the possibility of artifactual associations must be taken into account. Other genes currently under investigation are likely to be involved in $\mathrm{GH}$ and $\mathrm{PE}$ predisposition such as those associated with blood pressure control (Morgan and Ward 1999), thrombophilia (Kupferminc et al. 2000; Ozcan et al. 2001) and oxidative stress (Broughton Pipkin and Roberts 2000; Wang and Walsh 1996), but results are contradictory, possibly due to differences in study design, genetic heterogeneity of the disorder, the genetic background of the populations studied, as well as genetic stratification and type I error (GOPEC Consortium 2005). The French-Canadian population is characterized by a founder effect (Heyer and Tremblay 1995). It is expected to show little admixture and may be less exposed to false-positive results due to genetic stratification. Also, we obtained a very good recruitment rate $(85 \%)$, limiting the effect of possible bias. Generalization from this prospective study needs to be demonstrated.

Classically, GH is considered as a milder form of the disorder and has been much less extensively studied than PE. Although GH and PE may represent different manifestations of the same disease process, some evidence suggests that $\mathrm{GH}$, mild $\mathrm{PE}$ and severe PE may be pathophysiologically distinct entities (Brown et al. 2001; Fisher et al. 1981; Vatten and Skjaerven 2004). Our results suggest that $\mathrm{GH}$ and PE could represent different expressions of a spectrum of pathophysiological processes. Interestingly, previous work supported the hypothesis that $\mathrm{GH}$, rather than PE, was associated with insulin resistance (Caruso et al. 1999; Roberts et al. 1998). Furthermore, we (Forest et al. 2005) and others (Pouta et al. 2004) have previously observed that differences in fasting insulin concentrations between women with a past history of HDP and those with normal pregnancy were mostly due to a 10 $15 \%$ higher insulin concentration in those with prior GH compared with PE. We previously showed, in women in their mid-30s, that $25 \%$ of those with a past history of GH had an HDL-C $<1.00 \mathrm{mmol} / \mathrm{l}$ and triglycerides $\geq 2.0 \mathrm{mmol} / 1$ compared to $14 \%$ of those with prior PE (Forest et al. 2005). Genetic variations at the LIPC, LIPE and ApoCIII loci may partly explain the link between GH and CVD risk.

If they are confirmed, our findings strongly suggest that the combined effect of polymorphisms bearing modest individual susceptibility from independent genes involved in lipid metabolism and insulin action, namely ApoCIII, LIPC and LIPE genes, increases the likelihood of $\mathrm{GH}$ by 6- to 8-fold, favoring a distinct etiopathogeny between GH and PE. It is thus possible that $\mathrm{GH}-$ and PE-associated CVD risks later in life 
involve different pathophysiological pathways, but more studies of unrelated subjects with detailed questionnaires focusing on environmental exposure and life habits need to be undertaken to better identify genetic determinants predisposing women to $\mathrm{GH}$ and $\mathrm{PE}$, and possibly increasing their risk of CVD later in life.

Acknowledgments This work was supported by grants to Yves Giguère from the Fond de la Recherche en Santé du Québec (FRSQ) and to Jean-Claude Forest et al. from the Canadian Institutes of Health Research (CIHR). Joël Girouard is a fellow of the FRSQ, and Yves Giguère is a clinical investigator funded by the FRSQ. We thank Pastelle Ladouceur-Keigle, M.Sc., for her expertise in biostatistics.

\section{References}

Arngrimsson R, Bjornsson H, Geirsson R (1995) Analysis of different inheritance patterns in preeclampsia/eclampsia syndrome. Hypertens Pregnancy 14:27-38

Belo L, Gaffney D, Caslake M, Santos-Silva A, Pereira-Leite L, Quintanilha A, Rebelo I (2004) Apolipoprotein E and cholesteryl ester transfer protein polymorphisms in normal and preeclamptic pregnancies. Eur J Obstet Gynecol Reprod Biol 112:9-15

van Bockxmeer FM, Liu Q, Mamotte C, Burke V, Taylor R (2001) Lipoprotein lipase D9N, N291S and S447X polymorphisms: their influence on premature coronary heart disease and plasma lipids. Atherosclerosis 157:123-129

Broughton Pipkin F (2001) Risk factors for preeclampsia. N Engl J Med 344:925-926

Broughton Pipkin F, Roberts JM (2000) Hypertension in pregnancy. J Hum Hypertens 14:705-724

Brown MA, Lindheimer MD, de Swiet M, Van Assche A, Moutquin JM (2001) The classification and diagnosis of the hypertensive disorders of pregnancy: statement from the International Society for the Study of Hypertension in Pregnancy (ISSHP). Hypertens Pregnancy 20:IX-XIV

Caruso A, Ferrazzani S, De Carolis S, Lucchese A, Lanzone A, De Santis L, Paradisi G (1999) Gestational hypertension but not pre-eclampsia is associated with insulin resistance syndrome characteristics. Hum Reprod 14:219-223

Chappell LC, Seed PT, Briley A, Kelly FJ, Hunt BJ, CharnockJones DS, Mallet AI, Poston L (2002) A longitudinal study of biochemical variables in women at risk of preeclampsia. Am J Obstet Gynecol 187:127-136

Chen W, Srinivasan SR, Boerwinkle E, Berenson GS (2003) Hepatic lipase promoter C-514T polymorphism influences serial changes in HDL cholesterol levels since childhood: the Bogalusa Heart Study. Atherosclerosis 169:175-182

Chesley LC, Cooper DW (1986) Genetics of hypertension in pregnancy: possible single gene control of pre-eclampsia and eclampsia in the descendants of eclamptic women. $\mathrm{Br} \mathrm{J}$ Obstet Gynaecol 93:898-908

Chesley LC, Annitto JE, Cosgrove RA (1968) The familial factor in toxemia of pregnancy. Obstet Gynecol 32:303-311

Cooper D (1993) Hypertension in pregnancy. Marcel Dekker Inc., New York

Cooper DW, Brennecke SP, Wilton AN (1993) Genetics of preeclampsia. Hypertens Pregnancy 12:1-23

Dammerman M, Sandkuijl LA, Halaas JL, Chung W, Breslow JL (1993) An apolipoprotein CIII haplotype protective against hypertriglyceridemia is specified by promoter and 3' untranslated region polymorphisms. Proc Natl Acad Sci USA 90:4562-4566

Deeb SS, Peng R (2000) The C-514T polymorphism in the human hepatic lipase gene promoter diminishes its activity. J Lipid Res 41:155-158

Deeb SS, Zambon A, Carr MC, Ayyobi AF, Brunzell JD (2003) Hepatic lipase and dyslipidemia: interactions among genetic variants, obesity, gender, and diet. J Lipid Res 44:1279-1286

Dekker GA, Sibai BM (1998) Etiology and pathogenesis of preeclampsia: current concepts. Am J Obstet Gynecol 179:1359-1375

Efron B, Tibshirani RJ (1995) An introduction to the bootstrap. CRC Press, Boca Raton

Fisher KA, Luger A, Spargo BH, Lindheimer MD (1981) Hypertension in pregnancy: clinical-pathological correlations and remote prognosis. Medicine (Baltimore) 60:267276

Forest JC, Girouard J, Masse J, Moutquin JM, Kharfi A, Ness RB, Roberts JM, Giguere Y (2005) Early occurrence of metabolic syndrome after hypertension in pregnancy. Obstet Gynecol 105:1373-1380

Garenc C, Perusse L, Chagnon YC, Rankinen T, Gagnon J, Borecki IB, Leon AS, Skinner JS, Wilmore JH, Rao DC, Bouchard C (2002) The hormone-sensitive lipase gene and body composition: the HERITAGE Family Study. Int J Obes Relat Metab Disord 26:220-227

Garenc C, Aubert S, Laroche J, Girouard J, Vohl MC, Bergeron J, Rousseau F, Julien P (2004) Population prevalence of APOE, APOC3 and PPAR-alpha mutations associated to hypertriglyceridemia in French Canadians. J Hum Genet 49:691-700

GOPEC Consortium (2005) Disentangling fetal and maternal susceptibility for pre-eclampsia: a British multicenter candidate-gene study. Am J Hum Genet 77:127-131

Heyer E, Tremblay M (1995) Variability of the genetic contribution of Quebec population founders associated to some deleterious genes. Am J Hum Genet 56:970-978

Hubel CA, McLaughlin MK, Evans RW, Hauth BA, Sims CJ, Roberts JM (1996) Fasting serum triglycerides, free fatty acids, and malondialdehyde are increased in preeclampsia, are positively correlated, and decrease within 48 hours post partum. Am J Obstet Gynecol 174:975-982

Hubel CA, Roberts JM, Ferrell RE (1999) Association of preeclampsia with common coding sequence variations in the lipoprotein lipase gene. Clin Genet 56:289-296

Irion O, Masse J, Forest JC, Moutquin JM (1998) Prediction of pre-eclampsia, low birthweight for gestation and prematurity by uterine artery blood flow velocity waveforms analysis in low risk nulliparous women. $\mathrm{Br} \mathrm{J}$ Obstet Gynaecol 105:422-429

Ishimura-Oka K, Semenkovich CF, Faustinella F, Goldberg IJ, Shachter N, Smith LC, Coleman T, Hide WA, Brown WV, Oka K, et al. (1992) A missense (Asp250-Asn) mutation in the lipoprotein lipase gene in two unrelated families with familial lipoprotein lipase deficiency. J Lipid Res 33:745-754

Jansen H, Waterworth DM, Nicaud V, Ehnholm C, Talmud PJ (2001) Interaction of the common apolipoprotein C-III (APOC3 -482C > T) and hepatic lipase (LIPC $-514 \mathrm{C}>\mathrm{T}$ ) promoter variants affects glucose tolerance in young adults. European Atherosclerosis Research Study II (EARS-II). Ann Hum Genet 65:237-243

Jonsdottir LS, Arngrimsson R, Geirsson RT, Sigvaldason H, Sigfusson N (1995) Death rates from ischemic heart disease in women with a history of hypertension in pregnancy. Acta Obstet Gynecol Scand 74:772-776 
Kim YJ, Williamson RA, Chen K, Smith JL, Murray JC, Merrill DC (2001) Lipoprotein lipase gene mutations and the genetic susceptibility of preeclampsia. Hypertension 38:992-996

Klannemark M, Orho M, Langin D, Laurell H, Holm C, Reynisdottir S, Arner P, Groop L (1998) The putative role of the hormone-sensitive lipase gene in the pathogenesis of Type II diabetes mellitus and abdominal obesity. Diabetologia 41:1516-1522

Kupferminc MJ, Fait G, Many A, Gordon D, Eldor A, Lessing JB (2000) Severe preeclampsia and high frequency of genetic thrombophilic mutations. Obstet Gynecol 96:45-49

Levesque S, Moutquin JM, Lindsay C, Roy MC, Rousseau F (2004) Implication of an AGT haplotype in a multigene association study with pregnancy hypertension. Hypertension 43:71-78

Makkonen N, Heinonen S, Hiltunen M, Helisalmi S, Mannermaa A, Kirkinen P (2001) Apolipoprotein E alleles in women with pre-eclampsia. J Clin Pathol 54:652-654

Masse J, Forest JC, Moutquin JM, Marcoux S, Brideau NA, Belanger M (1993) A prospective study of several potential biologic markers for early prediction of the development of preeclampsia. Am J Obstet Gynecol 169:501-508

Masse J, Forest JC, Moutquin JM, Degrandpre P, Forest VI (1998) A prospective longitudinal study of platelet angiotensin II receptors for the prediction of preeclampsia. Clin Biochem 31:251-255

Morgan T, Ward K (1999) New insights into the genetics of preeclampsia. Semin Perinatol 23:14-23

NHBPEP Working Group (2000) Report of the national high blood pressure education program working group on high blood pressure in pregnancy. Am J Obstet Gynecol 183:S1S22

Ozcan T, Rinder HM, Murphy J, Kohn C, Copel JA, Magriples U (2001) Genetic thrombophilia and hypertensive complications of pregnancy. Obstet Gynecol 97:S40

Pihlajamaki J, Valve R, Karjalainen L, Karhapaa P, Vauhkonen I, Laakso M (2001) The hormone sensitive lipase gene in familial combined hyperlipidemia and insulin resistance. Eur J Clin Invest 31:302-308

Pouta A, Hartikainen AL, Sovio U, Gissler M, Laitinen J, McCarthy MI, Ruokonen A, Elliott P, Jarvelin MR (2004) Manifestations of metabolic syndrome after hypertensive pregnancy. Hypertension 43:1-7

Roberts JM (2000) Preeclampsia: what we know and what we do not know. Semin Perinatol 24:24-28

Roberts JM, Cooper DW (2001) Pathogenesis and genetics of pre-eclampsia. Lancet 357:53-56

Roberts RN, Traub AI, Kennedy AL, Hadden DR (1998) Glycosylated haemoglobin and hypertension arising in pregnancy. Br J Obstet Gynaecol 105:1122-1124

Roberts JM, Pearson G, Cutler J, Lindheimer M (2003) Summary of the NHLBI Working Group on research on hypertension during pregnancy. Hypertension 41:437-445

Salonen Ros H, Lichtenstein P, Lipworth L, Cnattingius S (2000) Genetic effects on the liability of developing pre-eclampsia and gestational hypertension. Am J Med Genet 91:256-260

Santé Québec (1995) Et la santé, comment ça va en 1992-1993? Rapport de l'enquête sociale et de santé 1992-1993. Minist- ère de la santé et des services sociaux, Gouvernement du Québec, Montreal

Seely EW, Solomon CG (2003) Insulin resistance and its potential role in pregnancy-induced hypertension. J Clin Endocrinol Metab 88:2393-2398

Sibai B, Dekker G, Kupferminc M (2005) Pre-eclampsia. Lancet 365:785-799

Skoglund-Andersson C, Ehrenborg E, Fisher RM, Olivecrona G, Hamsten A, Karpe F (2003) Influence of common variants in the CETP, LPL, HL and APO $\mathrm{E}$ genes on LDL heterogeneity in healthy, middle-aged men. Atherosclerosis 167:311-317

Solomon CG, Seely EW (2001) Brief review: hypertension in pregnancy: a manifestation of the insulin resistance syndrome? Hypertension 37:232-239

Tahvanainen E, Syvanne M, Frick MH, Murtomaki-Repo S, Antikainen M, Kesaniemi YA, Kauma H, Pasternak A, Taskinen MR, Ehnholm C (1998) Association of variation in hepatic lipase activity with promoter variation in the hepatic lipase gene. The LOCAT Study Invsestigators. J Clin Invest 101:956-960

Talmud PJ, Humphries SE (1997) Apolipoprotein C-III gene variation and dyslipidaemia. Curr Opin Lipidol 8:154-158

Talmud PJ, Palmen J, Walker M (1998) Identification of genetic variation in the human hormone-sensitive lipase gene and $5^{\prime}$ sequences: homology of $5^{\prime}$ sequences with mouse promoter and identification of potential regulatory elements. Biochem Biophys Res Commun 252:661-668

Talosi G, Endreffy E, Turi S, Nemeth I (2000) Molecular and genetic aspects of preeclampsia: state of the art. Mol Genet Metab 71:565-572

Thornton JG, Macdonald AM (1999) Twin mothers, pregnancy hypertension and pre-eclampsia. $\mathrm{Br} \mathrm{J}$ Obstet Gynaecol 106:570-575

Vatten LJ, Skjaerven R (2004) Is pre-eclampsia more than one disease? Bjog 111:298-302

Walker JJ (2000) Pre-eclampsia. Lancet 356:1260-1265

Wang Y, Walsh SW (1996) TNF alpha concentrations and mRNA expression are increased in preeclamptic placentas. J Reprod Immunol 32:157-169

Williams D (2003) Pregnancy: a stress test for life. Curr Opin Obstet Gynecol 15:465-471

Wilson BJ, Watson MS, Prescott GJ, Sunderland S, Campbell DM, Hannaford P, Smith WC (2003) Hypertensive diseases of pregnancy and risk of hypertension and stroke in later life: results from cohort study. BMJ 326:845

Zambon A, Deeb SS, Hokanson JE, Brown BG, Brunzell JD (1998) Common variants in promoter of the hepatic lipase gene are associated with lower levels of hepatic lipase activity, buoyant LDL, and higher HDL2 cholesterol. Arterioscler Thromb Vasc Biol 18:1723-1729

Zhang Q, Liu Y, Liu BW, Fan P, Cavanna J, Galton DJ (1998) Common genetic variants of lipoprotein lipase and apolipoproteins AI- CIII that relate to coronary artery disease: a study in Chinese and European subjects. Mol Genet Metab 64:177-183

Zhao JH, Curtis D, Sham PC (2000) Model-free analysis and permutation tests for allelic associations. Hum Hered 50:133-139 